\title{
Solving Second Order Linear Differential Equations with Klein's Theorem
}

\author{
M. van Hoeij*\& J.-A. Weil ${ }^{\dagger}$
}

\begin{abstract}
Given a second order linear differential equations with coefficients in a field $k=C(x)$, the Kovacic algorithm finds all Liouvillian solutions, that is, solutions that one can write in terms of exponentials, logarithms, integration symbols, algebraic extensions, and combinations thereof. A theorem of Klein states that, in the most interesting cases of the Kovacic algorithm (i.e when the projective differential Galois group is finite), the differential equation must be a pullback (a change of variable) of a standard hypergeometric equation. This provides a way to represent solutions of the differential equation in a more compact way than the format provided by the Kovacic algorithm. Formulas to make Klein's theorem effective were given in $[4,2,3]$. In this paper we will give a simple algorithm based on such formulas. To make the algorithm more easy to implement for various differential fields $k$, we will give a variation on the earlier formulas, namely we will base the formulas on invariants of the differential Galois group instead of semi-invariants.
\end{abstract}

\section{Categories and Subject Descriptors}

G.4 [Mathematical Software]: Algorithm design and analysis

\section{General Terms}

Algorithms

\section{Keywords}

Linear Differential Equations, Liouvillian solutions, Klein's Theorem

\author{
${ }^{*}$ Dept. of Mathematics, Florida State University, \\ Tallahassee, FL, USA. hoeij@math.fsu.edu \\ Supported by NSF grant 0098034 \\ ${ }^{\dagger}$ LACO, Université de Limoges, \\ 123 avenue Albert Thomas \\ 87060 LIMOGES CEDEX \\ jacques-arthur.weil@unilim.fr
}

Permission to make digital or hard copies of all or part of this work for personal or classroom use is granted without fee provided that copies are not made or distributed for profit or commercial advantage and that copies bear this notice and the full citation on the first page. To copy otherwise, to republish, to post on servers or to redistribute to lists, requires prior specific permission and/or a fee.

ISSAC'05, July 24-27, 2005, Beijing, China.

Copyright 2005 ACM 1-59593-095-705/0007 ...\$5.00.

\section{INTRODUCTION}

The Kovacic algorithm [19] computes closed form (Liouvillian) solutions of second order linear differential equations over $k=C(x)$. Since the appearance of [19], many papers have studied and refined the method. The version given in [27] uses invariants instead of the semi-invariants, which is easier to implement especially for differential fields $k$ more complicated than $C(x)$. The paper [15] gives good formulas for computing algebraic solutions (after [25]). The common basis of these algorithms is to derive solutions from (semi)invariants of the differential Galois group (see section 3).

Another approach is the Klein pullback method: Klein ([18], also $[1,5,2])$ showed that if the projective differential Galois group is finite, then the equation is a pullback of an equation in a finite list of well-known standard hypergeometric equations. This means that the solutions are of the form $e^{\int g} H(f)$ where $f, g \in k$ and $H$ is a standard hypergeometric function $H(x)={ }_{2} F_{1}([a, b],[c], x)$ whose parameters $a, b, c$ appear in a finite list. Interest in this method has recently been revived [5, 20, 21] for classifying work, but finding pullback functions still relied on skill.

In $[4,2,3]$ Berkenbosch and the authors of this paper give (surprisingly simple) formulas for computing the pullback function $f$ (as well as the function $g$ ). In $[2,3]$ Berkenbosch generalizes Klein's theorem to third order operators.

Our formulas from $[4,2,3]$ rely on computing semi-invariants of the differential Galois groups, which is well-mastered for differential equations with coefficients in $C(x)$. For more general differential fields, however, it may be easier (as noted in [27]) to use algorithms that compute invariants of the differential Galois group instead of semi-invariants. In order to use invariants, we will need to give formulas that are slightly different from those given in $[4,2,3]$.

The contribution in this paper is of algorithmic nature: we give an algorithm for solving second order differential by pullbacks for a general differential field $k$ by constructing new formulas which rely on invariants only. A field $k$ is admissible for our algorithm if:

$k$ is an effective (computable) field (this includes extracting square roots), one has an algorithm for computing rational solutions of linear differential equations with coefficients in $k$ and an algorithm for computing exponential solutions of second order differential equations.

Examples of admissible fields are Liouvillian extensions of $C(x)([24])$. Implementations of the above assumed algorithms are available for fields such as $C(x), C(x, \exp (f))$ ([8]), quadratic extensions of $C(x)([10])$, etc. For those 
fields $k$, the algorithm proposed here for computing Liouvillian solutions will be easy to implement.

Although we recall the main ideas in sections 3, we assume in this paper that the reader has an elementary knowledge of differential Galois theory ([23]) and of the Kovacic algorithm $[19,23]$. The algorithm in section 2 below follows the lines of the rational version of the Kovacic algorithm given in [27].

Section 2 contains the algorithm. Most of the remainder of the paper is devoted to its correctness and optional improvements. Section 3 contains material and definitions from differential Galois theory and Kovacic's algorithm; section 4 recalls the pullback formulas from $[4,2,3]$ for the case $k=C(x)$, Section 5 proves the pullback formulas for a general differential field and the correctness of the algorithm.

Finally, we remark that some recent papers $[7,12]$ showed how to solve certain classes of second linear differential equations as pullbacks of differential equations corresponding to special functions (Airy, Whittaker, etc). The present work is complementary to those whenever the differential equation has more than 3 singularities and the projective differential Galois group is not $\mathrm{PSL}_{2}$.

Acknowledgments. The authors would like to thank M. van der Put, M. Bronstein, M. Loday-Richaud and specially M. Berkenbosch for fruitful discussions while preparing this work. We thank the referees for their comments.

\section{THE ALGORITHM}

In this section, we state the algorithm assuming the reader is familiar with notations and concepts from differential Galois theory and Kovacic's algorithm; unfamiliar readers should proceed first to the next sections for explanations and come back to this section afterward.

Let $k$ denote a differential field of characteristic 0 . We consider the differential operator

$$
L=\partial^{2}+A_{1} \partial+A_{0} \in k[\partial]
$$

This corresponds to the differential equation $y^{\prime \prime}+A_{1} y^{\prime}+$ $A_{0} y=0$. We assume that there exists $w \in k$ such that $A_{1}=-\frac{w^{\prime}}{w}$ (this is not restrictive since after a simple transformation one may assume the stronger condition $A_{1}=0$, see section 3 ).

We define the following standard differential operators

$$
\begin{aligned}
S t_{D_{n}}^{s} & =\partial^{2}+\frac{x}{x^{2}-1} \partial-\frac{1}{4 n^{2}\left(x^{2}-1\right)}, n \in \mathbb{N} \\
S t_{\mathcal{G}}^{s} & =\partial^{2}+\frac{(8 x+3)}{6(x+1) x} \partial+\frac{(6 \nu-1)(6 \nu+1)}{144(x+1)^{2} x} \\
& \text { for }(\mathcal{G}, \nu) \in\left\{\left(A_{4}, 1 / 3\right),\left(S_{4}, 1 / 4\right),\left(A_{5}, 1 / 5\right)\right\} \\
S t_{D_{2}}^{i} & =\partial^{2}+\frac{4}{3} \frac{x}{\left(x^{2}-1\right)} \partial-\frac{5}{144} \frac{x^{2}+3}{\left(x^{2}-1\right)^{2}} \\
S t_{D_{n}}^{i} & =S t_{D_{n}}^{s}, n>2 \\
S t_{A_{4}}^{i} & =\partial^{2}+\frac{2\left(3 x^{2}-1\right)}{3 x\left(x^{2}-1\right)} \partial+\frac{5}{144 x^{2}\left(x^{2}-1\right)} \\
S t_{S_{4}}^{i} & =\partial^{2}+\frac{1}{4} \frac{(5 x-2)}{(x-1) x} \partial-\frac{7}{576} \frac{1}{(x-1)^{2} x}
\end{aligned}
$$

and $S t_{A_{5}}^{i}=S t_{A_{5}}^{s}$. These are well studied hypergeometric operators and their solutions are well-known. There are various ways to express the solutions of the above operators, one can use the hypergeometric function ${ }_{2} F_{1}$, or algebraic func- tions, or (if $\mathcal{G}$ is not $A_{5}$ ) nested radicals. We propose the ${ }_{2} F_{1}$ representation as the default choice because it is the most compact representation. Moreover, converting these ${ }_{2} F_{1}$ 's to algebraic functions or nested radicals is easier to implement (table lookup) than the reverse conversion.

The $m$-th symmetric power $L^{(ङ)}$ of $L$ is the operator whose solutions are spanned by products of $m$ solutions of $L$. Given differential operators $L \in k[\partial]$ and $\partial-b, b \in k$, the notation $L \otimes(\partial-b)$ refers to the operator whose solutions are the solutions of $L$ multiplied by the solution $e^{\int b}$ of $\partial-b$. Given a differential operator $\mathcal{L}=\partial^{2}+a_{1} \partial+a_{0}$, we define its $g$-invariant to be $g_{\mathcal{L}}:=2 a_{1}+\frac{a_{0}^{\prime}}{a_{0}}$.

We can now state the algorithm. The steps have to be performed in the given order, and the algorithm exits when a solution is found.

\section{Pullback Algorithm, general $k$ :}

Input: $L$ with $G(L) \subset \mathrm{SL}_{2}(C)$

Output: Liouvillian solutions, expressed via solutions of the above standard operators

1. Determine if $L$ has a solution $y$ such that $y^{\prime} / y \in k$ (an exponential solution). If so, return a basis of Liouvillian solutions of $L[15,2,19,27,23]$

2. Let $B_{4}$ be a basis of solutions in $k$ of $L^{(34}$

(a) If $B_{4}$ contains one element $i_{4}$, let $\partial^{2}+a_{1} \partial+a_{0}:=$ $L \otimes\left(\partial+\frac{i_{4}^{\prime}}{4 i_{4}}\right)$. Return $\sqrt[4]{i_{4}} e^{ \pm \int \sqrt{-a_{0}}}$ or use section 5.3 .

(b) (implementation of this step is optional). If $B_{4}$ contains two elements then let $m=6$ and take solutions as in step 3 below ( $B_{6}$ will have one element $i_{6}$ ), or use section 5.4.

3. For $m$ in $6,8,12$, let $B_{m}$ be a basis of solutions in $k$ of $L^{(s) m}$. If $B_{m}$ contains one element $i_{m}$, then let $\mathcal{L}=\partial^{2}+a_{1} \partial+a_{0}:=L \otimes\left(\partial+\frac{i_{m}^{\prime}}{m i_{m}}\right)$. Now return the following basis of solutions of $L$

$$
\sqrt[m]{i_{m}} H_{1}(f), \quad \sqrt[m]{i_{m}} H_{2}(f)
$$

where $H_{1}(x), H_{2}(x)$ is a basis of solutions of $S t_{\mathcal{G}}^{i}$ and where $\mathcal{G}$ and $f$ are determined as follows:

(a) If $m=6$, then $\mathcal{G}:=A_{4}$ and $f:=\sqrt{1+\frac{64}{5} \frac{a_{0}}{g_{\mathcal{L}}^{2}}}$. This $f$ will be in $k$.

(b) If $m=8, \mathcal{G}:=S_{4}$ and $f=-\frac{7}{144} \frac{g_{\mathcal{L}}^{2}}{a_{0}}$.

(c) If $m=12, \mathcal{G}:=A_{5}$ and $f=\frac{11}{400} \frac{g_{\mathcal{L}}^{2}}{a_{0}}$.

The name of the standard operators refers to the projective differential Galois group $P G(L)$ (see section 3 below) of $L$.

4. Otherwise the operator has no Liouvillian solutions.

The above algorithm is correct but improvements are possible. In step $2 \mathrm{a}$ where $B_{4}$ has one element, we have $P G(L)=$ $D_{n}$ for some $n>2$. If an integration algorithm for the field $k\left(\sqrt{-a_{0}}\right)$ is available, then we could use it to try to simplify the expression $e^{ \pm \int \sqrt{-a_{0}}}$. However, if $n \neq \infty$ then there is an alternative that is likely to be more efficient. To implement this alternative, one starts by running a subroutine of the integration algorithm ([6]) that determines $n$. When 
$n$ is found, if $n \neq \infty$, then instead of running the remainder of the integration algorithm one proceeds by using the formulas in section 5.4 .

Implementation of step $2 \mathrm{~b}$ is optional. In step $2 \mathrm{~b}$, the projective Galois group is $D_{2}$ (this denotes $C_{2} \times C_{2}$ ). If step $2 \mathrm{~b}$ is not implemented, then in the $D_{2}$ case the algorithm will proceed to step $3 \mathrm{a}$ and compute solutions using formulas meant for $A_{4}$. Although these formulas give correct solutions for the $D_{2}$ case (note that $D_{2} \triangleleft A_{4}$ and that these two groups have the same invariants of degree 6) one can find better (more compact) solutions in this case by using equation (2.4) and the formula from section 5.4.

\section{DIFFERENTIAL GALOIS THEORY}

For completeness and to set notations, we briefly recall the rational Kovacic algorithm from [27]. Let $L=\partial^{2}+A_{1} \partial+A_{0}$ where $A_{0}, A_{1} \in k$. We consider a second order ordinary linear differential equation

$$
L y=0, \quad y^{\prime \prime}+A_{1} y^{\prime}+A_{0} y=0 .
$$

We assume that $A_{1}=\frac{f^{\prime}}{f}$ for some $f \in k$; this can be achieved after a change of variable $y \mapsto y e^{\int \frac{A_{1}}{2}}$ which turns the equation (3.8) into the reduced form $y^{\prime \prime}-r y=0$ with $r=\frac{A_{1}^{2}}{4}+\frac{A_{1}^{\prime}}{2}-A_{0}$.

Given two linearly independent solutions of (3.8), say $y_{1}, y_{2}$ (either "formal" or "actual functions on some open set"), the field $K:=k\left(y_{1}, y_{2}, y_{1}^{\prime}, y_{2}^{\prime}\right)$ is a differential field (a field closed under differentiation) and is generated, as a differential field, by $y_{1}$ and $y_{2}$ over $k$. This field $K$ is called a Picard-Vessiot extension of (3.8). The solution space in $K$ is the $C$ vector space generated by $y_{1}$ and $y_{2}$, denoted by $V$ in all that follows. The group of differential automorphisms of $K$ over $k$ (i.e., automorphisms of $K$ over $k$ that commute with $\partial$ ) is called the differential Galois group of (3.8) over $k$. We denote it by $G(L)=\operatorname{Gal}_{K / k}(L)$. The condition $A_{1}=\frac{f^{\prime}}{f}$ ensures that $G(L) \subset \mathrm{SL}_{2}(C)$.

The projective Galois group is defined by

$$
P G(L):=G(L) /\left(G(L) \cap C^{*}\right),
$$

where $G(L) \cap C^{*}$ denotes the subgroup of those $g \in G$ that act on $V$ as scalar multiplication.

Multiplying the solutions by $e^{\int b}$ for $b$ in $k$ changes the Galois group $G(L)$ but not the projective Galois group $P G(L)$. The operator whose solutions are $y \cdot e^{\int b}$, with $y$ solution of $L(y)=$ 0 , is denoted $L \otimes(\partial-b)$. We will say that two operators $L_{1}$ and $L_{2}$ are projectively equivalent when there exists $b \in k$ such that $L_{1}=L_{2} \otimes(\partial-b)$. It is easy to see that $L_{1}$, $L_{2}$ are projectively equivalent if and only if they have the same reduced form. If $L_{1}, L_{2}$ are projectively equivalent then $P G\left(L_{1}\right)=P G\left(L_{2}\right)$.

\subsection{Invariants and Semi-Invariants}

The key to Kovacic's algorithm is that the existence of Liouvillian solutions is (for second order equations) equivalent with the existence of a semi-invariant of the differential Galois group.

DeFinition 3.1. Fix a basis $y_{1}, y_{2}$ of the solution space $V$ of $L$.

1. A homogeneous polynomial $I\left(Y_{1}, Y_{2}\right) \in C\left[Y_{1}, Y_{2}\right]$ is called an invariant with respect to the differential operator $L$ if its evaluation $h:=I\left(y_{1}, y_{2}\right)$ is invariant under the action of the differential Galois group $G(L)$ of $L$. In other words $h \in k$. This function $h$ is then called the value of the invariant polynomial $I$.

2. A homogeneous polynomial $I\left(Y_{1}, Y_{2}\right) \in C\left[Y_{1}, Y_{2}\right]$ is called a semi-invariant with respect to a differential operator $L$ if $\frac{h^{\prime}}{h} \in k$ where $h:=I\left(y_{1}, y_{2}\right)$.

We will list a few well known facts, for more details see $[25,23,19]$. For second order operators, there is a one to one correspondence between the (semi)-invariants of degree $m$ and their values (for higher order operators this need not be the case). The values of invariants of degree $m$ are precisely the rational solutions of $L^{(5)}$, i.e solutions in $k$. The values of the semi-invariants of degree $m$ are the so-called exponential solutions of $L^{(s)}$, that is, those solutions $h$ of $L^{(3) m}$ for which $h^{\prime} / h \in k$.

The operator $L^{(s m}$ can be easily computed from the recursion given in (1.14) in [11] (see also [9]): Let $L_{0}=1, L_{1}=\partial$ and

$$
L_{i+1}=\left(\partial+i A_{1}\right) L_{i}+i(m-(i-1)) A_{0} L_{i-1}
$$

for $0<i \leq m$, then $L_{m+1}=L^{(3) m}$.

\subsection{The Subgroups of $\mathrm{SL}_{2}(C)$}

Invariants and semi-invariants are elements of $C\left[Y_{1}, Y_{2}\right]$. In the algorithm we will not calculate the invariants themselves, but only their values. For each semi-invariant, we will only compute the logarithmic derivative $h^{\prime} / h$ of the value $h$ of a semi-invariant. So in the following, when we write that there are $n$ semi-invariants of degree $m$, we are counting the number of distinct $h^{\prime} / h \in k$ for which $h$ is a solution of $L^{(s m}$. And when we write that there are $n$ invariants of degree $m$, we mean that the set of solutions of $L^{\Im m}$ in $k$ has a basis with $n$ elements.

We recall the classification of subgroups of $\mathrm{SL}_{(} C$ ) (see e.g $[19,25,27,23])$ and the invariants and semi-invariants of lowest degree. The group is reducible if there is at least one invariant line in $V$. A non-zero element of that line is an exponential solution, i.e., a solution whose logarithmic derivative is in $k$ (see $[23,27,15,2]$ for more on this case). The rest of the classification (irreducible cases) is in the above references:

LEMMA 3.2 (IMPRIMITIVE GROUPS). Assume that $G(L) \subset S L_{2}(C)$ and that $G(L)$ is imprimitive, i.e. irreducible and there exist two lines $l_{1}, l_{2} \subset V$ such that $G(L)$ acts on $\left\{l_{1}, l_{2}\right\}$ by permutation. Then $P G(L) \subset D_{\infty}$ (infinite dihedral group). Three cases are to be considered.

1. $P G(L)=D_{2}$. Three semi-invariants $S_{2, a}, S_{2, b}, S_{2, c}$ of degree $2\left(S_{2, x}^{2}\right.$ is invariant), two invariants $I_{4, a}, I_{4, b}$ of degree 4. One invariant $I_{6}$ of degree 6 , with $I_{6}=$ $S_{2, a} S_{2, b} S_{2, c}$. Note that the notation $D_{2}$ does not refer to the cyclic group $C_{2}$ but to $C_{2} \times C_{2}$.

2. $P G(L)=D_{n}, n>2$. One semi-invariant $S_{2}$ of degree 2, one invariant $I_{4}=S_{2}^{2}$ of degree 4, and another invariant $I_{2 n}$ of degree $2 n$.

3. $P G(L)=D_{\infty}$ has only one semi-invariant $S_{2}$ of degree 2 and one invariant $I_{4}=S_{2}^{2}$ of degree 4. 
Lemma 3.3 (Primitive Groups). Assume $G$ is primitive, i.e neither reducible nor imprimitive, and $G(L) \subset$ $S L_{2}(C)$. Four cases are to be considered.

1. $P G(L)=A_{4}$; two semi-invariant $S_{4, a}, S_{4, b}$ of degree 4 , one invariant $I_{6}$ of degree 6 , and one invariant $I_{8}$ of degree 8 , with $I_{8}=S_{4, a} S_{4, b}$

2. $P G(L)=S_{4}$; one semi-invariant $S_{6}$ of degree 6 , one invariant $I_{8}$ of degree 8.

3. $P G(L)=A_{5}$; one invariant $I_{12}$ of degree 12 .

4. $G=S L_{2}(C)$; no semi-invariants and no Liouvillian solutions.

The degrees for the (semi)-invariants of these groups allow to give a list of possible symmetric powers $L^{\text {(S) }}$ to investigate. This is the key to the Kovacic algorithm (semiinvariants) or its Ulmer-Weil rational variant [27] (invariants). Computing invariants (or semi-invariants), one can find the type of the differential Galois group (a little more needs to be done to discriminate $D_{n}$ from $D_{\infty}$, see section 4.4). We summarize this in the following immediate corollary

COROLlary 3.4. In the Pullback algorithm from section 2, in the case of step 1 the group is reducible, in case of step $2 a$ the projective Galois group is $D_{\infty}$ or some $D_{n}, n>2$. It is $D_{2}$ in case of step $2 b, A_{4}$ in step $3 a, S_{4}$ in step $3 b, A_{5}$ in step $3 c$, and $\mathrm{PSL}_{2}$ otherwise.

For each possible finite projective group, pullback formulas can be computed; this is done in the next section.

\section{PULLBACK FORMULAS, CASE $K=C(X)$}

In this section, we recall our work with Maint Berkenbosch from $[4,2]$. The next subsection is standard material $[1,2$, $5,20,21]$

\subsection{Standard equations}

If $y_{1}, y_{2}$ is a basis of solutions of $L$, then define $C_{L}:=$ $C\left(\frac{y_{1}}{y_{2}}\right)$, which is a subfield of the Picard-Vessiot extension $K$. The field $C_{L}$ does not depend on the choice of basis (replacing $y_{1}, y_{2}$ by another basis corresponds to a Möbius transformation of $\left.\frac{y_{1}}{y_{2}}\right)$. Replacing $y_{1}, y_{2}$ by $e^{\int v} y_{1}, e^{\int v} y_{2}$ for some function $v$ does not affect $C_{L}$ either. In fact, given two operators $L_{1}$ and $L_{2}$, one has $C_{L_{1}}=C_{L_{2}}$ if and only if $L_{1}$ and $L_{2}$ are projectively equivalent.

The projective Galois group $P G(L)$ acts faithfully on $C_{L}$. The field $C_{L}^{P G(L)}$ of invariants under this action can, by Luroth's theorem, be written as $C(f)$ for some $f \in k$. We say that an operator $S t$ is a standard equation for $P G(S t)$ if $C_{S t}^{P G(S t)}$ equals $C(z)$ for some $z$ with $z^{\prime}=1$.

Now assume that $L$ has projective group $P G$ and $S t$ is a standard equation with projective Galois group $P G$. If $C_{L}^{P G}=C(f)$, then $z \mapsto f$ maps $C_{S t}^{P G}$ to $C_{L}^{P G}$. This, and the fact that $C_{L}$ determines $L$ up to projective equivalence, are key ideas in Klein's theorem below. Before stating this, we set a family of standard equations. All other standard equations can then be found using Möbius $x \mapsto(a x+b) /(c x+d)$ and projective equivalence $L \mapsto L \otimes(\partial+v)$ transformations.
A standard equation for each finite projective differential Galois group can be found among the hypergeometric equations

$$
S t_{P G}=\partial^{2}+\frac{a}{x^{2}}+\frac{b}{(x-1)^{2}}+\frac{c}{x(x-1)}
$$

where the coefficients $a, b, c$ are related to the differences $\lambda, \mu, \nu$ of the exponents at 0,1 , and $\infty$ by the relations

$$
a=\frac{1-\lambda^{2}}{4} \quad b=\frac{1-\mu^{2}}{4} \quad \text { and } \quad c=\frac{1-\nu^{2}+\lambda^{2}+\mu^{2}}{4} .
$$

More precisely, one can choose $(\lambda, \mu, \nu)=\left(\frac{1}{2}, \frac{1}{2}, \frac{1}{n}\right)$ for $P G=D_{n},\left(\frac{1}{3}, \frac{1}{2}, \frac{1}{3}\right)$ for $P G=A_{4},\left(\frac{1}{3}, \frac{1}{2}, \frac{1}{4}\right)$ for $P G=S_{4}$ and $\left(\frac{1}{3}, \frac{1}{2}, \frac{1}{5}\right)$ for $P G=A_{5}$.

The index $P G$ refers to the projective differential Galois group of $S t_{P G}$ corresponding to the chosen values of $a, b, c$. These equations and their solutions are well known.

\subsection{Klein's theorem}

Definition 4.1. Let $L_{1} \in C(z)\left[\frac{d}{d z}\right]$ and $L_{2} \in k[\partial]$ be linear differential operators.

1. $L_{2}$ is a proper pullback of $L_{1}$ by $f \in k$ if the change of variable $z \mapsto f$ changes $L_{1}$ into $L_{2}$.

2. $L_{2}$ is a (weak) pullback of $L_{1}$ by $f \in k$ if there exists $v \in k$ such that $L_{2} \otimes(\partial+v)$ is a proper pullback of $L_{1}$ by $f$.

TheOREM 4.2 (KLein, $[18,1,2])$. Let $L$ be a second order irreducible linear differential operator over $k$ with projective differential Galois group $P G(L)$. Then, $P G(L) \in$ $\left\{D_{n}, A_{4}, S_{4}, A_{5}\right\}$ if and only if $L$ is a (weak) pullback of $S t_{P G(L)}$.

Let $L$ have a projective differential Galois group $P G(L)$ and suppose the standard equation with projective differential Galois group $P G(L)$ has $H_{1}, H_{2}$ as a $C$-basis of solutions. The theorem of Klein says that $L$ is a pullback of $S t_{P G(L)}$. Suppose we know $f$ and $v$ as in definition 4.1, then a $C$-basis of solutions of $L y=0$ is given by $H_{1}(f) e^{\int v}$ and $H_{2}(f) e^{\int v}$.

$H_{1}$ and $H_{2}$ are known for all standard equations. To get the solutions in explicit form one should then determine the projective differential Galois group and, in case it is finite, determine $f$ and $v$. It was remarked in $[1,5]$ (and somehow in [18]) that $f$ can be expressed as a quotient of invariants of the differential Galois group, but this idea was not used algorithmically. We will build $f$ (and $v$ ) using semi-invariants in section 4 , and using invariants in section 5 .

The difficulty lies in the fact that $L$ is a weak pullback of a standard equation, i.e it is only projectively equivalent to a proper pullback of the standard equation. The key to formulas is to compute a normal form such that the normal form of $L$ will be a proper pullback of its standard form.

Suppose that $L$ has a differential Galois group $G$ (and projective group $P G$ ) with semi-invariant $S$ of degree $m$ and value $\sigma$. And suppose the value of $S$ with respect to the standard operator $S t_{P G}$ equals $\sigma_{0}$ (modulo $C^{*}$ ). Then, the value of $S$ w.r.t. both the differential operator $S_{G}=S t_{P G} \otimes$ $\left(\partial_{z}+\frac{\sigma_{0}^{\prime}}{m \sigma_{0}}\right)$ and the differential operator $\mathcal{L}=L \otimes\left(\partial_{x}+\frac{\sigma^{\prime}}{m \sigma}\right)$ is equal to 1 and the following property holds. 
Lemma 4.3. $\mathcal{L}$ is a proper pullback of $S_{G}$.

Proof. The (semi)-invariant of $S_{G}$ corresponding to $\sigma$ (in the above notations) has value 1 so it is mapped to 1 under any pullback transformation $z \mapsto f$. $L$ is a weak pullback by Klein's theorem, so $L \otimes(\partial-v)$ will be a proper pullback for some $v$; but its (semi)-invariant is $e^{\int m v}$, which should be 1 , so $v$ must be 0 and hence $L$ must be a proper pullback.

A direct examination (and relevant choices of standard equations) in each case will provide the pullback function $f$.

\subsection{Formulas: the primitive case}

The projective Galois group is in $\left\{A_{4}, S_{4}, A_{5}\right\}$ in this section. The standard equation in reference is $S t_{P G} y=0$ where the differences of exponents are $\lambda=\frac{1}{3}$ at $x=0$, $\mu=\frac{1}{2}$ at $x=1$, and $\nu=\frac{1}{3}$ for $A_{4}, \frac{1}{4}$ for $S_{4}$ and $\frac{1}{5}$ for $A_{5}$ at $x=\infty$.

The differential Galois group of this equation has a semiinvariant $S$ of degree $m=4$ in the case of $A_{4}$, degree $m=6$ in the case of $S_{4}$ and $m=12$ in the case of $A_{5}$ with value $\sigma_{0}(x)=x^{-m / 3}(x-1)^{-m / 4}$. The new equation $S_{G}=S t_{P G} \otimes$ $\left(\partial+\frac{1}{3 x}+\frac{1}{4(x-1)}\right)$ now has an invariant of degree $m$ with value 1 . Rearranging it (via a Möbius transform, to obtain nicer formulas), we get the normalized standard equation:

$$
S t_{P G}^{s}:=\partial^{2}+\frac{1}{6} \frac{(8 x+3)}{(x+1) x} \partial+\frac{s}{(x+1)^{2} x}
$$

with $s=\frac{(6 \nu-1)(6 \nu+1)}{144}$ (recall that $\nu$ is $\frac{1}{3}, \frac{1}{4}, \frac{1}{5}$ for cases $A_{4}, S_{4}, A_{5}$ respectively). It has exponents $\left(\frac{\nu}{2}+\frac{1}{12},-\frac{\nu}{2}+\frac{1}{12}\right)$ at $-1,\left(0, \frac{1}{2}\right)$ at 0 and $\left(0, \frac{1}{3}\right)$ at $\infty$ where $\nu$ has the previous value in each case.

LEMMA 4.4. Let $\mathcal{L}=\partial^{2}+a_{1} \partial+a_{0}$ be a normalized operator with $P G(\mathcal{L}) \in\left\{A_{4}, S_{4}, A_{5}\right\}$ (i.e it has an invariant of degree $m$ with value 1 for the above values of $m$ ). Define $g_{\mathcal{L}}:=2 a_{1}+\frac{a_{0}^{\prime}}{a_{0}}$. Then $\mathcal{L}$ is a proper pullback of $S t_{P G}^{s}$ and the pullback mapping is

$$
f:=9 s \frac{g_{\mathcal{L}}^{2}}{a_{0}}
$$

Proof. Lemma 4.3 shows that $\mathcal{L}$ is a proper pullback $z \mapsto f$ of $S t_{P G}^{s}$ for some $f$. Computing this pullback and equating it to $\mathcal{L}$ gives the relations $a_{1}=\frac{f^{\prime}}{2 f}+\frac{5 f^{\prime}}{6(f+1)}-\frac{f^{\prime \prime}}{f^{\prime}}$ and $a_{0}=\frac{s f^{\prime 2}}{(f+1)^{2} f}$ whence $\frac{a_{0}^{\prime}}{a_{0}}=-\frac{2 f^{\prime}}{f+1}-\frac{f^{\prime}}{f}+\frac{2 f^{\prime \prime}}{f^{\prime}}$ and the formula follows by simple elimination.

In fact, the formula was not obtained that way: as we know that $\mathcal{L}$ is a proper pullback and that the solution $f$ is unique (by Klein's theorem and our normalization), we compute the expression of the image of $S t_{P G}^{s}$ under a generic pullback and perform differential elimination $[13,14]$ (there are other ways to find the formula but this way was the least amount of work). In the same way one can obtain formulas for other choices of standard equations but those turn out to be larger.

So, given $L=\partial^{2}+A_{1} \partial+A_{0}$ with finite primitive projective group, the pullback function is found the following way:

Pullback for $A_{4}, S_{4}, A_{5}$, semi-invariant version Input: $L=\partial^{2}+A_{1} \partial+A_{0}$ with $P G(L) \in\left\{A_{4}, S_{4}, A_{5}\right\}$. Output: Pullback function $f$.
1. For $m \in\{4,6,12\}$ check for a semi-invariant of degree $m$ and call $v$ its logarithmic derivative.

2. If yes, the projective group $P G(L)$ is known. Let $\mathcal{L}=$ $L \otimes\left(\partial+\frac{1}{m} v\right)$; this is a proper pullback of $S t_{P G}^{s}$ with invariant value 1 .

3. Write $\mathcal{L}=\partial^{2}+a_{1} \partial+a_{0}$. Compute $g_{\mathcal{L}}:=2 a_{1}+\frac{a_{0}^{\prime}}{a_{0}}$, and the pullback mapping is $f:=9 s \frac{g_{\mathcal{L}}^{2}}{a_{0}}$

REMARK 4.5. The change of variable $z \mapsto f$ changes $g_{S t}$ to $g_{S t}(f) \cdot f^{\prime}$. Now, $g_{S t}=-\frac{1}{3(x+1)}$ and the relation $g_{\mathcal{L}}=$ $-\frac{f^{\prime}}{3(f+1)}$ yields another method to find $f$. This approach will fail for imprimitive groups because then $g_{\mathcal{L}}$ will be zero.

\subsection{Formulas: the imprimitive case}

In this case, the projective Galois group is $P G(L)=D_{n}$ for $n \in \mathbb{N}$. To simplify formulas, here, we choose the standard equation with exponent differences $\frac{1}{2}$ at +1 and -1 and $\frac{1}{n}$ at infinity. It has a semi-invariant $S_{2}=Y_{1} Y_{2}$ of degree 2 and two semi-invariants $S_{n, a}=Y_{1}^{n}+Y_{2}^{n}$ and $S_{n, b}=Y_{1}^{n}-Y_{2}^{n}$ of degree $n$. The chosen standard equation

$$
S t_{D_{n}}^{s}=\partial^{2}-\frac{z}{z^{2}-1} \partial-\frac{1}{4 n^{2}} \frac{1}{z^{2}-1}
$$

has exponents $\left(0, \frac{1}{2}\right)$ at +1 and -1 and $\left(\frac{-1}{2 n}, \frac{1}{2 n}\right)$ at $\infty$; it has a semi-invariant of degree 2 and value 1 .

An operator $\mathcal{L}=\partial^{2}+a_{1} \partial+a_{0}$ is a proper pullback of $S_{D_{n}}$ if $a_{0}=-\frac{1}{4 n^{2}} \frac{f^{\prime 2}}{f^{2}-1}$ and $a_{1}=-\frac{1}{2} \frac{a_{0}^{\prime}}{a_{0}}$. The equation $\mathcal{L} y=0$ admits the solutions $y_{1}, y_{2}=\exp \int \pm \sqrt{-a_{0}}$ i.e. $y_{1}=\sqrt[2 n]{f+\sqrt{f^{2}-1}}$ and $y_{2}=1 / y_{1}$. The number $n$ can thus be determined with (a subroutine of) the algorithm of elementary integration ([6]) applied to $\sqrt{-a_{0}}$.

For $N \in \mathbb{N}$, the expressions $y_{1}^{N}$ and $y_{2}^{N}$ are permuted by the Galois group and are found to be a basis of solutions of $\mathcal{L}_{N}:=\partial^{2}+a_{1} \partial+N^{2} a_{0}$. In particular $L_{2 n}$ has solutions $f$ (rational) and $\sqrt{f^{2}-1}$. Once $n$ is known, we would like to compute $f$ from a rational solution $F$ of $L_{2 n}$. However, we would only know it up to a constant so we use its logarithmic derivative:

Lemma 4.6. Let $\mathcal{L}=\partial^{2}+a_{1} \partial+a_{0}$ be an irreducible operator with an invariant of degree 2 with value 1. Assume that $P G(\mathcal{L})=D_{n}$. Let $F$ be a rational solution of $\partial^{2}+a_{1} \partial+4 n^{2} a_{0}$ and let $u:=\frac{F^{\prime}}{F}$. Then the solutions of $\mathcal{L}$ are $y_{1}=\sqrt[2 n]{f+\sqrt{f^{2}-1}}$ and $y_{2}=\sqrt[-2 n]{f+\sqrt{f^{2}-1}}$ with $f=\sqrt{\frac{1}{1+\frac{u^{2}}{4 n^{2} a_{0}}}}$.

Proof. By the above discussion, $\partial^{2}+a_{1} \partial+4 n^{2} a_{0}$ has a rational solution and $F=c f$ for some constant $f$. Now we have $f^{\prime 2}=-4 n^{2} a_{0}\left(f^{2}-1\right)$. Dividing out by $f^{2}$ yields the formula.

REMARK 4.7. Despite the square root in the expression of $f$, the function is rational. However, if the constant field of $k$ is not algebraically closed, a quadratic extension of the constants may be needed in computing this square root (see also [2, 16] and references therein). 
Pullback Formula for $D_{n}$, semi-invariant version Input: $L=\partial^{2}+A_{1} \partial+A_{0}$ with $P G(L)=D_{n}$ ( $n$ unknown). Output: Pullback function $f$ and the solutions.

1. Compute a semi-invariant of degree 2 and compute its logarithmic derivative $v$.

2. If yes, let $\mathcal{L}=L \otimes\left(\partial+\frac{1}{2} v\right)$; it is a proper pullback of $S_{D_{n}}$ with invariant value 1 .

3. Denote $\mathcal{L}=\partial^{2}+a_{1} \partial+a_{0}$. Determine a candidate for (a multiple of) $n$. (note: if there is more than one semi-invariant of degree 2 , then $n=2$ )

4. Compute a rational solution $F$ of $\mathcal{L}_{n}:=\partial^{2}+a_{1} \partial+$ $4 n^{2} a_{0}$ and let $u=\frac{F^{\prime}}{F}$.

5. Return the solutions $y_{1}=e^{\int \frac{v}{2} \sqrt[2 n]{f+\sqrt{f^{2}-1}}}$ and $y_{2}=e^{\int \frac{v}{2} \sqrt[-2 n]{f+\sqrt{f^{2}-1}}}$ with $f=\sqrt{\frac{1}{1+\frac{u^{2}}{4 n^{2} a_{0}}}}$.

\section{PULLBACK FORMULAS, GENERAL $K$}

\subsection{Standard Equations}

The algorithm for general $k$ uses only invariants (not semiinvariants). Hence, the relevant normal form for the standard and target equations will be the one for which an appropriate invariant (often one with the lowest degree) has value 1. For a projective group $P G$, a standard equation with semi-invariant of lowest degree with value 1 (resp. with invariants of lowest degree value 1) will be denoted $S t_{P G}^{s}$ (resp. $\left.S t_{P G}^{i}\right)$.

A second idea that we will use is the fact that $D_{2} \subset A_{4} \subset S_{4}$. So, a standard equation for $D_{2}$ (resp. $A_{4}$ ) is a pullback of some $S t_{A_{4}}$ (resp. $S t_{S_{4}}$ ). Transformations between those equations can be found in [26] (or can be recomputed, as below).

Like in the previous section, we will proceed in reverse order of the classification to give the pullback formulas

\subsection{Primitive Cases}

\subsubsection{Icosaedral case $A_{5}$}

The group is determined by an invariant of degree 12 , as in the $C(x)$ case, so we use the formula from section 4.3 .

\subsubsection{Octaedral case $S_{4}$}

Let $S t_{S_{4}}^{s}$ denote the standard equation from section 4.3 with projective Galois group $S_{4}$. It has an invariant of degree 6 with value 1 . However our target differential operator $L$ has $G(L) \subset S L_{2}$. It only has a semi-invariant $S_{6}$ of degree 6 and an invariant $I_{8}$ of degree 8 . Having computed the value of the (semi)-invariant of degree 8 of $S t_{S_{4}}^{s}$, we tensor $S t_{S_{4}}^{s}$ with $\partial-\frac{1}{24(x+1)}$ (and, via a Möbius transform, change the singularities to 0,1 and $\infty$ to simplify the formula of lemma 5.1) to obtain the standard operator

$$
S t_{S_{4}}^{i}=\partial^{2}+\frac{1}{4} \frac{(5 x-2)}{(x-1) x} \partial-\frac{7}{576} \frac{1}{(x-1)^{2} x}
$$

Its exponents are $\left(0, \frac{1}{2}\right)$ at $0,\left(-\frac{1}{24}, \frac{7}{4}\right)$ at 1 , and $\left(0, \frac{1}{4}\right)$ at $\infty$; it has an invariant of degree 8 with value 1 .

We assume that the differential operator $L$ has projective Galois group $S_{4}$ and $G(L) \subset \mathrm{SL}_{2}(C)$. Thus $L$ has an invariant of degree 8 with value $\sigma$. We normalize $L$ by tensoring with $\partial+\frac{\sigma^{\prime}}{8 \sigma}$ so its normal form has an invariant of degree 8 with value 1 .

LEMmA 5.1. Let $\mathcal{L}=\partial^{2}+a_{1} \partial+a_{0} \in k[\partial]$ be a normalized differential operator with projective Galois group $P G(\mathcal{L})=$ $S_{4}(\mathcal{L}$ is normalized to have an invariant of degree 8 with value 1$)$. Define $g_{\mathcal{L}}:=2 a_{1}+\frac{a_{0^{\prime}}}{a_{0}}$. Then $\mathcal{L}$ is a proper pullback of $\mathrm{St}_{S_{4}}^{i}$ and the pullback mapping is

$$
f=-\frac{7}{144} \frac{g_{\mathcal{L}}^{2}}{a_{0}}
$$

Proof. That $\mathcal{L}$ is a proper pullback of $S t_{S_{4}}^{i}$ follows from lemma 4.3. Pick an unknown function $f$ and form the change of variable $x=f$ in $S t_{S_{4}}^{i}$. We obtain $a_{0}=-\frac{7}{576} \frac{f^{\prime 2}}{(f-1)^{2} f}$ and $a_{1}=-\frac{f^{\prime \prime}}{f^{\prime}}+\frac{1}{2} \frac{f^{\prime}}{f}+\frac{3}{4} \frac{f^{\prime}}{f-1}$ Performing standard differential elimination on the latter, see $[13,14]$ and references therein, yields the above formula.

With this formula, the algorithm in section 4.3 is straightforward to adapt (compute an invariant of degree 8 of $L$ instead of a semi-invariant of degree 6).

\subsubsection{Tetrahedral case $A_{4}$}

Let $S t_{A_{4}}^{s}$ denote the standard equation from section 4.3 with projective Galois group $A_{4}$. It has an invariant of degree 4 with value 1 . As $G(L) \subset S L_{2}(C)$, our $L$ has only semi-invariants in degree 4 , but it has an invariant in degree 6. So, proceeding as in section 5.2.2 (with lemma 3.3.1 in mind) yields a new standard operator $S t_{A_{4}}^{i}$ for $A_{4}$ with an invariant of degree 6 having value 1 :

$$
S t_{A_{4}}^{i}=\partial^{2}+\frac{2\left(3 x^{2}-1\right)}{3 x\left(x^{2}-1\right)} \partial+\frac{5}{144} \frac{1}{x^{2}\left(x^{2}-1\right)}
$$

Its exponents are $\left(0, \frac{1}{3}\right)$ at 1 and -1 , and $\left(-\frac{1}{12}, \frac{5}{12}\right)$ at 0 (the point $\infty$ is non-singular).

We assume that the differential operator $L$ has projective Galois group $A_{4}$ and $G(L) \subset \mathrm{SL}_{2}(C)$. Thus $L$ has an invariant of degree 6 with value $\sigma$. We normalize $L$ by tensoring with $\partial+\frac{\sigma^{\prime}}{6 \sigma}$ so the resulting normal form $\mathcal{L}$ has an invariant of degree 6 with value 1 .

LeMMA 5.2. Let $\mathcal{L}=\partial^{2}+a_{1} \partial+a_{0} \in k[\partial]$ be a normalized differential operator with projective Galois group $P G(L)=$ $A_{4}$, i.e $L$ has an invariant of degree 6 with value 1 . Then $\mathcal{L}$ is a proper pullback of $S t_{A_{4}}^{i}$. Let $g_{\mathcal{L}}:=2 a_{1}+\frac{a_{0^{\prime}}}{a_{0}}$. Then the pullback mapping is

$$
f= \pm \sqrt{1+\frac{64}{5} \frac{a_{0}}{g_{\mathcal{L}}^{2}}}
$$

Proof. One can use the same differential elimination argument as for lemma 5.1. Note that Klein's theorem shows that $1+\frac{64}{5} \frac{a_{0}}{g^{2}}$ must be the square of an element of $k$.

REMARK 5.3. The appearance of a square-root is no surprise because the standard equation for $A_{4}$ has a symmetry (exchange 1 and -1 ) so there are two solutions to the pullback problem (see [16, 2] and references therein), each "attached" to one of the two semi-invariants of degree 4. In the algorithm in section 4.3 we need to choose one of the two semi-invariants, hence the (apparent) uniqueness of the pullback formula there. 
An alternative approach to find and prove the formula in the lemma 5.2 is the following. As $L$ is a pullback of $S t_{A 4}^{i}$, it is also a pullback of $S t_{S_{4}}^{i}$ because $A_{4} \subset S_{4}$. Now apply the $S_{4}$ formula to the $A_{4}$ standard equation, solve, and one obtains lemma 5.2. The same idea can also be used for $D_{2}$.

\subsection{Dihedral Groups $D_{n}, n>2$}

The case $P G(L) \subset D_{\infty}$ is characterized by the existence of an invariant $I_{4}$ of degree 4 . We assume that $P G(L) \neq D_{2}$ so the space of invariants of degree 4 has dimension 1 (and $I_{4}$ is the square of a semi-invariant of degree 2). Tensoring $L$ with $\partial+\frac{I_{4}^{\prime}}{4 I_{4}}$, we obtain a normalized operator $\mathcal{L}$ which has an invariant of degree 2 with value 1 . So we can use the algorithm from section 4.4 (start at step 3) and obtain the pullback function.

REMARK 5.4. The difficulty in this subsection lies in deciding whether $P G(L)$ is some $D_{n}$ or $D_{\infty}$. Computing $n$ is achieved by computing the torsion of some divisor from the integration algorithm, which can be achieved under our assumptions on $k$, see [6] or [2, 3].

\subsection{Quaternion Group $D_{2}$}

There is a problem to choose a relevant normalization because the space of invariants of degree 4 is two-dimensional and, in our normalizations, we would need to choose one among those that is a square of a semi-invariant of degree 2 in order to use the formulas from section 4.4. Although this is possible (e.g [27]), we propose a few simpler approaches (the reader is welcome to select whichever one she likes best). As $G(L) \subset \mathrm{SL}_{2}(C)$, the operator has a unique (up to constants) invariant of degree 6 with value $\sigma$ (the product of the three semi-invariants of degree 2 ). Tensoring $L$ with $\partial+\frac{\sigma^{\prime}}{6 \sigma}$, we obtain a normalized operator $\mathcal{L}$ whose invariant of degree 6 has value 1 .

Approach 1: We have $D_{2} \subset A_{4}$. Moreover, $\mathcal{L}$ has an invariant of degree 6 with value 1 . So $\mathcal{L}$ is a proper pullback of $S t_{A_{4}}^{i}$ from section 5.2.3 and the pullback is computed directly with the algorithm from section 5.2.3. The good point is that no work is needed; the bad point is that the solutions will be given in terms of the solutions of $S t_{A_{4}}^{s}$ which is not very good if, for example, we want the minimal polynomial or an expression by radicals.

Approach 2: In approach 1, we have computed a pullback $F$ from $S t_{A_{4}}^{i}$ so solutions of $\mathcal{L}$ are $\widetilde{H}_{i}(F)$ with $\widetilde{H}_{i}$ solutions of $S t_{A_{4}}^{i}$. Now we precompute the pullback from $D_{2}^{i}$ to $A_{4}^{i}$. First send singularities to $0,1, \infty$ by a Möbius transform; next, tensor by a first order operator so that the exponents are $(0,1 / 3)$ at 0 and $\infty$. Changing $x$ to $x^{3}$, the preimages of 0 and $\infty$ will have exponents $(0,1)$ so they will be ordinary, while the preimages of 1 (i.e $1, j, j^{2}$ ) will have exponent differences $1 / 2$ : the resulting equation is thus a standard $D_{2}$ equation. Sending the singularities to $-1,1, \infty$ and tensoring by a first order operator finally sends us to the standard operator $S t_{D_{2}}$. We find that $\widetilde{H}_{i}\left(\frac{3 \sqrt{-3}\left(x^{2}-1\right)}{x^{3}-9 x}\right)=H_{i}(x)$ with $H_{i}$ solutions of $S t_{D_{2}}^{i}$. So the solutions of $\mathcal{L}$ will be $H_{i}(f)$ where $f$ is a root of the third degree equation

$$
\left(3 \sqrt{-3}\left(f^{2}-1\right)\right)-F\left(f^{3}-9 f\right)=0
$$

By Klein's theorem, the latter has three roots $f$ in $k$ which can be computed, e.g by factoring the above. We note that, because the solution is not unique, factoring is inevitable in this process.

\section{CONCLUSION}

TheOREM 6.1. The algorithm of section 2 is correct.

Proof. The steps compute the projective Galois group by [27] or corollary 3.4. Step 2a is sections 5.3 and 4.4; Step $2 \mathrm{~b}$ is sections 5.4 and 4.4; Step 3a is section 5.2.3; Step 3b is section 5.2.2; and Step 3c is sections 5.2.1 and 4.3.

The algorithm presented here is very easy to implement for an admissible differential field. Further improvements and speedups can be provided in the case when $k=C(x)$. The algorithm is implemented in Maple 9.5. A draft implementation (and a maple worksheet to check most formulas of this paper) can be consulted at http://www.unilim.fr/ pages_perso/jacques-arthur.weil/issac05/

Denote $H(x)={ }_{2} F_{1}([-1 / 60,11 / 60],[2 / 3], 1 /(x+1))$ which is one of the solutions of $S t_{A_{5}}^{s}$. The Kovacic algorithm produces the minimal polynomial $m_{K}$ of $y^{\prime} / y$ for some solution $y$ of $S t_{A_{5}}^{s}$, whereas Fakler's algorithm [15] produces the minimal polynomial $m_{F}$ of a solution $y$ of $S t_{A_{5}}^{s}$. Note that $m_{F}$ is preferable over $m_{K}$.

Now consider the following example: $L=48 x(x-1)(75 x-$ 139) $\partial^{2}+\left(2520 x^{2}-47712 x / 5+3336\right) \partial-19 x+36001 / 75$ which has projective Galois group $A_{5}$. The pullback function $f$ is rather large (the degree is 31 ). By default our algorithm uses hypergeometric functions to denote the answer. In essence this means that $x$ in the expression $H(x)$ above is being replaced by $f$. To get a solution of $L$ in the same format as would have been produced by Kovacic's resp. Fakler's algorithm, one essentially has to substitute $f$ for $x$ in the solution that these algorithms provided for $S t_{A_{5}}^{s}$. However, this substitution will lead to a large expression because $x$ occurs many times in the expression $m_{K}$ resp. $m_{F}$ and all those occurrences are replaced by $f$. We compared the kovacicsols command in Maple 9.5 (which follows the usual Kovacic algorithm) with the algorithm presented here. The size of the output (measured with the command length) in Maple 9.5 was 236789 whereas for the new algorithm the size is only 1360. Note that this new algorithm is scheduled to appear in the kovacicsols command in the next version of Maple.

\section{REFERENCES}

[1] Baldassarri, F; Dwork, B: Differential Equations with Algebraic Solutions, American Journal of Mathematics 101, 1979, p42-76.

[2] Berkenbosch, M: Algorithms and Moduli Spaces for Differential Equations $\mathrm{PhD}$ dissertation, Rijksuniversiteit Groningen, 2004.

[3] Berkenbosch, M: Pullbacks for Differential Equations, submitted

[4] Berkenbosch, M; van Hoeij, M; Weil, J-A: Recent Algorithms for Solving Second-Order Differential Equations summary by Michele Loday-Richaud. INRIA research report $\sharp 5003$. Algorithms seminar, 2001-2002 http: //algo.inria.fr/seminars/sem01-02/weil.pdf

[5] Beukers, F; van der Waall, A: Lamé equations with algebraic solutions. J. Differential Equations 197 (2004), no. 1, 1-25 
[6] Bronstein, M: Integration of elementary functions. J. Symbolic Comput. 9 (1990), no. 2, 117-173.

[7] Bronstein, M ; Lafaille, S: Solutions of linear ordinary differential equations in terms of special functions. Proceedings of the 2002 International Symposium on Symbolic and Algebraic Computation, 23-28, ACM, New York, 2002.

[8] Bronstein, M; Fredet, A: Solving linear ordinary differential equations over $C\left(x, e^{\int f(x) d x}\right)$. Proceedings of the 1999 International Symposium on Symbolic and Algebraic Computation (Vancouver, BC), 173-179 , ACM, New York, 1999.

[9] Bronstein, M; Mulders, T; Weil, J-A: On symmetric powers of differential operators. Proceedings of the 1997 International Symposium on Symbolic and Algebraic Computation, 156-163, ACM, New York, 1997.

[10] Burger R, van Hoeij M, and Labahn G: Closed Form Solutions of Linear Odes having Elliptic Function Coefficients. International Symposium on Symbolic and Algebraic Computation'04 Proceedings, 58-64, ACM, New York, 2004

[11] Chalkley, R: Relative invariants for homogeneous linear differential equations, J. Differential Equations 80, 107-153, 1989.

[12] Chan, L; Cheb-Terrab, E.S: Non-liouvillian solutions for second order Linear ODEs. International Symposium on Symbolic and Algebraic Computation'04 Proceedings, 80-86, ACM, New York, 2004

[13] Hubert, E: Notes on triangular sets and triangulation-decomposition algorithms II: Differential Systems, in Symbolic and Numerical Scientific Computing 2630 p. 40-87 (Ed: Winkler, F. and Langer, U.), Springer Verlag Heidelberg, 2003

[14] Boulier, F; Hubert, E: DIFFALG: description, help pages and examples of use Symbolic Computation Group, University of Waterloo, Ontario, Canada, 1998. Now at http:

//www.inria.fr/cafe/Evelyne.Hubert/diffalg
[15] Fakler, W: On second order homogeneous linear differential equations with Liouvillian solutions. Computer algebra (Saint-Louis, 1996). Theoret. Comput. Sci. 187 (1997), no. 1-2, 27-48.

[16] Hendriks, P.A.; van der Put, M: Galois action on solutions of a differential equation. J. Symbolic Comput. 19 (1995), no. 6, 559-576.

[17] van Hoeij, Mark: The Minimum Polynomial of an Algebraic Solution of Abel's problem. Preprint FSU00-02.

[18] Klein, F: Ueber lineare Differentialgleichungen, Math. Ann. 12 (1877) 167-179.

[19] Kovacic, J.J: An Algorithm for Solving Second Order Linear Differential Equations, Journal of Symbolic Computation 2, 1986, p3-43.

[20] Liţcanu, R: Lamé operators with finite monodromy-a combinatorial approach. J. Differential Equations 207 (2004), no. 1, 93-11

[21] Maier, R.S: Algebraic solutions of the Lam equation, revisited. J. Differential Equations 198 (2004), no. 1, 16-34

[22] van der Put, M: Galois Theory of Differential Equations, Algebraic Groups and Lie Algebras, Journal of symbolic computation 28, 1999, p441-472.

[23] van der Put, M: Singer, M.F: Galois Theory of linear Differential Equations, Grundlehren der mathematischen Wissenschaften, Springer 2003.

[24] Singer, M.F: Liouvillian Solutions of Linear Differential Equations with Liouvillian Coefficients Journal of Symbolic Computation, 1991.

[25] Singer, M.F ; Ulmer, F: Liouvillian and algebraic solutions of second and third order linear differential equations. J. Symbolic Comput. 16 (1993), no. 1, 37-73.

[26] Vidunas, R: Algebraic transformations of Gauss hypergeometric functions, preprint (2004) http://arxiv.org/abs/math.CA/0408269

[27] Ulmer, F ; Weil, J-A Note on Kovacic's algorithm. J. Symbolic Comput. 22 (1996), no. 2, 179-200. 